\title{
Editorial
}

International Archives of
Allergy
Immunology

\section{Twin Epidemics: Asthma and Obesity}

\author{
Brian D. Kent Stephen J. Lane \\ Department of Respiratory Medicine, Adelaide and Meath Hospital, Tallaght, Ireland
}

As a rising tide of obesity engulfs the developed world, so the list of diseases associated with being overweight lengthens. While there is a decades-long appreciation of the association between excess fat mass and cardiovascular and metabolic disease, recent years have seen the identification of a link between obesity and other noncommunicable comorbidities, including cancer, venous thromboembolic disease and asthma. Evidence for an independent relationship between asthma and obesity comes from a series of large epidemiological studies, with cross-sectional data generally demonstrating asthma prevalence increasing with body mass [1]. Meanwhile, a recent meta-analysis of prospective studies suggested a dose-dependent increased risk of incident asthma in overweight and obese populations [2]. Indeed, obese asthma may be considered a distinct clinical phenotype, characterised by later onset, female preponderance and greater symptomatology than classic atopic asthma, and by a relatively low degree of eosinophilic inflammation [3].

The vast bulk of this data relates to western (mainly Caucasian) populations, however, and little data exist examining the interaction between overweight, obesity and asthma in Asian subjects. Consequently, the work of Fukutomi et al. [4] in this issue of the International Archives of Allergy and Immunology is both important and timely. Using a validated questionnaire of respiratory symptoms and medication use, the authors examined the prevalence of asthma in a nationwide Japanese population of nearly 23,000 individuals. Stratifying the results by body mass index (BMI), and using logistic regression analysis to adjust for the effects of age, smoking status, allergic rhinitis and pet ownership, the authors found a striking J-shaped relationship between BMI and both asthma prevalence and respiratory symptoms. Using subjects with a BMI of 18.5-22.9 as the referent group, a statistically significant increase in asthma prevalence was observed among men with a BMI of $\geq 27.5$ and among women with a BMI of $\geq 23$. Moreover, this increased risk appeared dose-dependent, with both men and women with a BMI of $\geq 30$ having an odds ratio of greater than 3 for a diagnosis of asthma compared with the referent group. Notably, similar effects were seen in participants under and over the age of 45 .

If one accepts the concept of obesity being a driver of asthma, these results could in many respects be considered unsurprising. Japanese populations appear to have a greater percentage of body fat at a given BMI than western subjects, and appear at increased risk of cardiovascular and metabolic disease at lower BMIs than Caucasians

\section{KARGER}

Fax +4161306 1234 E-Mail karger@karger.ch www.karger.com
() 2011 S. Karger AG, Basel

$1018-2438 / 12 / 1573-0213 \$ 38.00 / 0$

Accessible online at:

www.karger.com/iaa
Correspondence to: Dr. Brian D. Kent

Pulmonary and Sleep Disorders Unit

St. Vincent's University Hospital

Dublin 4 (Ireland)

Tel. +353 1221 3702,E-Mail brian.kent@ ucd.ie 
[5]. Nonetheless, the increase in the risk of asthma at even very modestly elevated BMI is striking, and serves to underline the difficulty in the global application of the findings of studies of European and North American populations.

The relationship between asthma and obesity provides a fertile area for research. Existing data suggest that concomitant obesity and the metabolic syndrome may play a contributory role in asthma pathogenesis via a number of mechanisms [6]. Excess abdominal and chest wall fat mass can mechanically compromise respiratory function, reducing functional residual capacity, tidal volumes and tidal bronchodilatation. Meanwhile, adipose tissue in obese subjects is dysfunctional and inflamed, secreting increased concentrations of pro-inflammatory adipokines such as tumour necrosis factor- $\alpha$ and leptin, with a parallel reduction in production of the anti-inflammatory peptide adiponectin. These immunological changes have been demonstrated to contribute to asthmatic inflammation in mice, although studies in human subjects have yielded conflicting results [7]. Finally, hyperglycaemia and hyperinsulinaemia resulting from insulin resistance may promote airway smooth muscle dysfunction, goblet cell metaplasia, and subepithelial fibrosis [8]. Whether these putative mechanisms are pertinent in obese Asian asthmatics remains unclear.

While this study has several important strengths, including a large population sample, it has a number of important limitations. By their very nature, studies reliant on self-reported outcome data are susceptible to respond- er bias, and while the European Community Respiratory Health Survey questionnaire utilized in the study is well validated in European populations, its reliability outside of this context is uncertain. Similarly, although cross-sectional, observational studies such as this may usefully identify associations, they provide little information on causality or mechanisms of association. It would also have been fascinating had some additional information on asthma medication usage among the different weight categories been available for analysis. Not only would this have provided some internal validation of asthma diagnoses, it may also have allowed some evaluation of the influence of weight on asthma severity in an Asian population - an area ripe for exploration. Finally, as the authors themselves appropriately note, confounding factors not incorporated into their statistical model - e.g. air pollution or concomitant illness - may have contributed to the effects seen.

In conclusion, although the work of Fukutomi et al. [4] clearly cannot allow any major mechanistic insights, it certainly suggests that the ongoing research effort examining the relationship between asthma and obesity has major relevance for Asian as well as European and North American populations. It also adds to the growing evidence base demonstrating the inadequacy of western definitions of overweight and obesity in Asian populations. That this paper perhaps gives rise to as many questions as it answers, should, we believe, be viewed as a strength rather than a weakness.
References
Ford ES: The epidemiology of obesity and asthma. J Allergy Clin Immunol 2005;115: 897-909, quiz 10.

2 Beuther DA, Sutherland ER: Overweight, obesity, and incident asthma: a meta-analysis of prospective epidemiologic studies. Am J Respir Crit Care Med 2007;175:661-666.

-3 Haldar P, Pavord ID, Shaw DE, et al: Cluster analysis and clinical asthma phenotypes. Am J Respir Crit Care Med 2008;178:218224.

4 Fukutomi Y, Taniguchi M, Nakamura H, Konno S, Nishimura M, Kawagishi Y, Okada C, Tanimoto Y, Takahashi K, Akasawa A, Akiyama K: Association between body mass index and asthma among Japanese adults: risk within the normal weight range. Int Arch Allergy Immunol 2012;157: 281-287.
WHO Expert Consultation: Appropriate body-mass index for Asian populations and its implications for policy and intervention strategies. Lancet 2004;363:157-163.

-6 Shore SA, Fredberg JJ: Obesity, smooth muscle, and airway hyperresponsiveness. J Allergy Clin Immunol 2005;115:925-927.

7 Sood A: Obesity, adipokines, and lung disease. J Appl Physiol 2010;108:744-753.

8 Agrawal A, Mabalirajan U, Ahmad T, Ghosh B: Emerging interface between metabolic syndrome and asthma. Am J Respir Cell Mol Biol 2011;44:270-275. 